\title{
Corela
}

Cognition, représentation, langage

HS-5 | 2006

Organisation des textes et cohérence des discours

\section{Quand le texte signale sa structure : la fonction textuelle des noms sous-spécifiés}

\section{Dominique Legallois}

\section{(2) OpenEdition}

\section{Journals}

\section{Édition électronique}

URL : http://journals.openedition.org/corela/1465

DOI : $10.4000 /$ corela. 1465

ISSN : $1638-573 \mathrm{X}$

\section{Éditeur}

Cercle linguistique du Centre et de l'Ouest - CerLICO

\section{Référence électronique}

Dominique Legallois, « Quand le texte signale sa structure : la fonction textuelle des noms sousspécifiés », Corela [En ligne], HS-5 | 2006, mis en ligne le 27 octobre 2006, consulté le 19 avril 2019. URL : http://journals.openedition.org/corela/1465; DOI : 10.4000/corela.1465

Ce document a été généré automatiquement le 19 avril 2019

\section{(c) (i) (2)(2)}

Corela - cognition, représentation, langage est mis à disposition selon les termes de la licence Creative Commons Attribution - Pas d'Utilisation Commerciale - Partage dans les Mêmes Conditions 4.0 International. 


\section{Quand le texte signale sa structure : la fonction textuelle des noms sous- spécifiés}

Dominique Legallois

Nous nous intéressons dans cet article à deux phénomènes linguistiques, en soi indépendants l'un de l'autre, mais qui peuvent dans certaines configurations, être articulés: les structures d'enchaînement et les noms sous-spécifiés. Il semble que la linguistique textuelle a sous-estimé le fonctionnement de ces phénomènes dans la structuration et l'organisation des discours. Aussi s'agit-il d'apporter ici un premier éclairage sur ce fonctionnement, qui appellera nécessairement d'autres développements, tant le thème est complexe. Un point terminologique s'impose avant d'aller plus en avant: structures d'enchaînement (désormais $\mathrm{SE}$ ) désigne des configurations générales, globales, à partir desquelles se déploie le texte, ou une partie du texte ; par ex : [PROBLÈME - SOLUTION] [DÉSIR - ASSOUVISSEMENT] [OBJECTIF - RÉALISATION]. La partie I est consacrée à la présentation et à la justification de la fonctionnalité de ces structures. Noms sous-spécifiés désigne une catégorie nominale assez hétérogène, mais qui est formellement identifiable par le fait que ses membres entrent tous dans la composition des énoncés spécificationnels, c'est-à-direles constructions avec complément phrastique : [N être de + infinitif] ou [N être que + P conjonctive] ; par exemple :

1- Pardonne-moi, Raymond. C'est encore les lentilles. Tu sais pourquoi. Le malheur est qu'on ne peut pas trouver du persil en cette saison. (G. Duhamel, Le Notaire du Havre, 1933).

2- La société, étant divisée par tranches comme un bambou, la grande affaire d'un homme est de monter dans la classe supérieure à la sienne, et tout l'effort de cette classe est de l'empêcher de monter. (Stendhal, Souvenirs d'égotisme, 1832)

2 La partie II introduit les spécificités syntaxiques, sémantiques, et textuelles des noms sous-spécifiés. La partie III souligne la complémentarité entre structures d'enchaînement et un ensemble de noms sous-spécifiés: dans un nombre important de textes, en 
particulier expositifs / argumentatifs, les structures d'enchaînement sont souvent signalées au moyen de ce type de noms.

A côté de la dimension référentielle des noms, de leur ouverture sur "l'extérieur textuel ", cette étude voudrait souligner une autre dimension généralement attribuée aux seuls connecteurs : le rôle de mise en relation de différentes parties du texte.

\section{I les structures d'enchaînement}

\section{Introduction}

$4 \quad$ Il est possible d'établir très schématiquement une hiérarchie des modes organisationnels du texte. Au sommet, le genre discursif assure la relation d'un texte particulier à une formation socio-discursive, à un interdiscours; les genres, en principe infinis car dépendants de l'activité humaine, se divisent en de nombreux sous-genres. A un échelon plus bas, le type, ou plutôt, pour suivre J. M. Adam (1992), la séquence textuelle, à la fois réseau relationnel hiérarchique et entité autonome dotée d'une organisation interne : la séquence « narrative », « descriptive», " argumentative ", « explicative » et « dialogale ». On notera avec J.M. Adam le fait qu'un texte dont le statut est bien identifié, emprunte généralement à plusieurs de ces séquences (par exemple un récit relève principalement de la séquence "narrative», mais aussi de la séquence "descriptive », « argumentative ») - témoignage de l'hétérogénéité compositionnelle du texte. A un dernier niveau, les relations de cohérence (ou de discours) entre propositions, phrases, groupes de phrases, qui ont souvent retenu l'attention des linguistiques depuis quelques années, dans la mesure où est impliqué l'ensemble des questions de connexité, d'anaphoricité, d'enchaînements transphrastiques et de relations logiques ${ }^{1}$.

5 Cette échelle à trois niveaux est bien sûr présentée ici de façon très schématique ; elle ne porte que sur les dispositifs de mise en relation (liage) et non sur les aspects référentiels et énonciatifs. Nous faisons l'hypothèse d'ajouter à ces paliers, un quatrième niveau, nommé, dans ce travail, structure d'enchaînement ${ }^{2}$, qui viendrait s'intégrer entre les relations de cohérence et les séquences. En effet, l'examen des séquences textuelles montre que celles-ci, malgré leurs différences, partagent des aspects communs. Ainsi, le schéma ci-dessous emprunté à J. M. Adam, permet de visualiser les éléments compositionnels de la séquence narrative mis en évidence par les travaux sémiotiques et narratologiques :

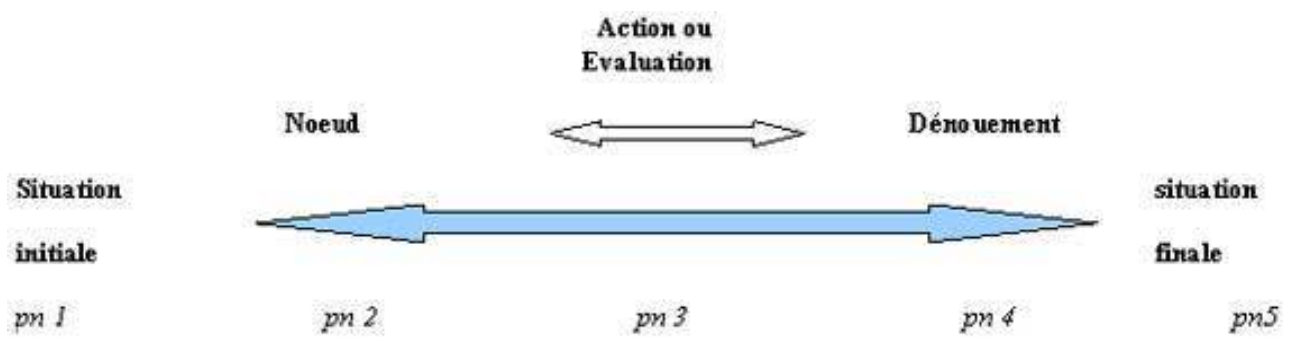

Figure 1 : Schéma de la séquence narrative d'après Adam $1992: 54$

Par ailleurs, la séquence explicative prototypique est composée de 


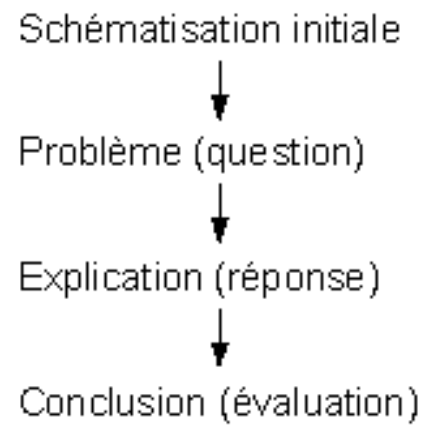

Figure 2: Schéma de la séquence explicative adapté d'Adam 1992: 132

7 Ces deux types de séquences engendrent des textes dont la nature, fort différente, est discriminée sans difficulté par les locuteurs. Pourtant, il est possible d'établir assez facilement une relation entre les deux types: tous les deux sont des réalisations distinctes d'une même structure plus générale. Ainsi, situation et schématisation initiale relèvent d'une catégorie permettant la présentation d'un état premier; nœud et problème actualisent une complication, dénouement et explication proposent des solutions ou des réponses; conclusion et état final élaborent une situation finale. Il y a donc bien une structure sousjacente commune à ces deux types séquentiels (mais aussi à la séquence argumentative) : la structure d'enchaînement (SE) ; cette remarque n'a évidemment rien d'original, puisque Hutchins (1977) notait déjà la parenté organisationnelle des récits et les textes scientifiques $^{3}$; nous reprenons donc l'idée que les articulations discutées naguères par Brémond (1973) ou Greimas (1966) pour le récit, peuvent sans aménagement spectaculaire être appliquées à d'autres types de textes. Ces articulations, constituant des formes "simples ${ }^{4}$, variables, déformables, font preuve, par instanciation dans diverses séquences, d'une productivité souvent relevée par la didactique, mais négligée par la linguistique du texte ${ }^{5}$ lorsque celle-ci s'arrête à des considérations trop locales.

\section{Caractérisation}

8 A un niveau général, les structures d'enchaînement mettent en jeu une situation et l'évaluation de cette situation, qui donne souvent lieu à un problème, à une complication, à un dilemme ou à une question à résoudre, un objectif à réaliser, un désir à combler, une connaissance à acquérir. Ou bien encore, sans que la complication soit le fruit d'un jugement évaluatif, elle surgit en tant qu'événement, donc indépendamment de l'intentionnalité des sujets, et ainsi déséquilibre la situation initiale. Il faut donc attribuer à ces structures des réalisations différentes, qui obligent à une caractérisation quelque peu floue. Par ailleurs, [PROBLÈME - SOLUTION], [DÉSIR - SATISFACTION], [OBJECTIF - RÉALISATION], [QUESTION - RÉPONSE] $]^{6}$, etc. peuvent être perçus comme des réalisations différentes d'un même schéma cognitif global, vague, hautement productif : un objectif est toujours un "problème » dont il faut chercher la "réponse » dans la réalisation; de même, tout problème ou toute complication a pour vocation d'être dépassé dans une solution. Même le couple bien assorti [QUESTION - RÉPONSE] ne résiste pas à la présence d'un tiers. Comme l'a montré récemment A. Berrendonner, le rapport question / réponse, dans un dialogue, n'est pas une simple relation binaire : répondre consiste à réparer un état inconsistant du savoir partagé (Berrendonner, 2005 :169). Autrement dit, réparer constitue la solution à un 
manque informatif (problème) perçu dans la mémoire discursive commune. Ce qui est évalué comme problématique dans ce cas, est la déficience de connaissances communes, c'est-à-dire, non pas un élément rapporté par le texte, mais une condition fondamentale à la communication.

Cependant, les réalisations textuelles des SE ne doivent pas dissimuler leurs dimensions cognitives, sémiotiques, et anthropologiques. Les structures d'enchainement sont sûrement efficientes aux niveaux les plus fondamentaux; le pédiatre D. Stern fait l'hypothèse, en ce qui concerne le developpement cognitif de l'enfant de moins d'un an, de la constitution d'enveloppes pré-narratives. Les enveloppes pré-narratives sont des unités de base hypothétiques de la vie psychique du nourrisson, qui se définissent par une proto-intrigue comportant un agent, une action, un but, un objet, un contexte. Ainsi, l'unité DÉSIR - MOTIVATION (pour nous, une "structure d'enchaînement ») apparaît, par exemple, à chaque fois que l'enfant a faim; le bébé expérimente alors le scénario PROBLÈME - SOLUTION; ses pleurs déclenchent l'interaction avec ses parents qui apporteront la «solution». Dans cette petite histoire qui se répète plusieurs fois quotidiennement, l'enfant construit une structure narrative cohérente, possédant une dimension émotionnelle et dramatique, se déroulant dans le temps, et qui est enrichie à chaque expérience. Se construisent de cette façon, par abstractions successives, des patterns prototypiques organisant d'autres types d'expériences :

11 Exemple 1

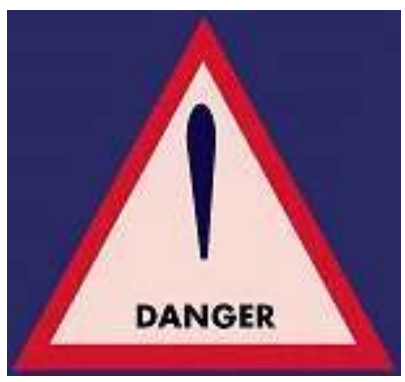

http://www.palais-decouverte.fr/discip/astro/venus/images/Panneau3venus-danger.jpg

Ce panneau signale une situation évaluée comme dangereuse, donc reconnue comme problématique, dont la solution pour l'automobiliste (codée institutionnellement par le règlement de la circulation routière, donc une solution "déontique ») est de ralentir. Tout sujet est confronté quotidiennement à ce type de situations qui demande un comportement soit automatique et à peine conscient, soit, au contraire, une adaptation extrêmement réfléchie.

On peut légitimement suspecter la qualité humoristique de la "blague » ci-dessous (circulant sur Internet), mais, comme de nombreuses blagues ou devinettes, elle repose de façon exemplaire sur le ressort [PROBLÈME - SOLUTION] :

Exemple $2^{7}$

\section{Enigme}

17 Vous êtes en Afrique. 2 On vous a tendu un piège. 3 Vous êtes attaché en haut d'un arbre par une corde. 3' Une bougie au sol est en train de brûler la corde. 4 Voici le résumé de la situation : 


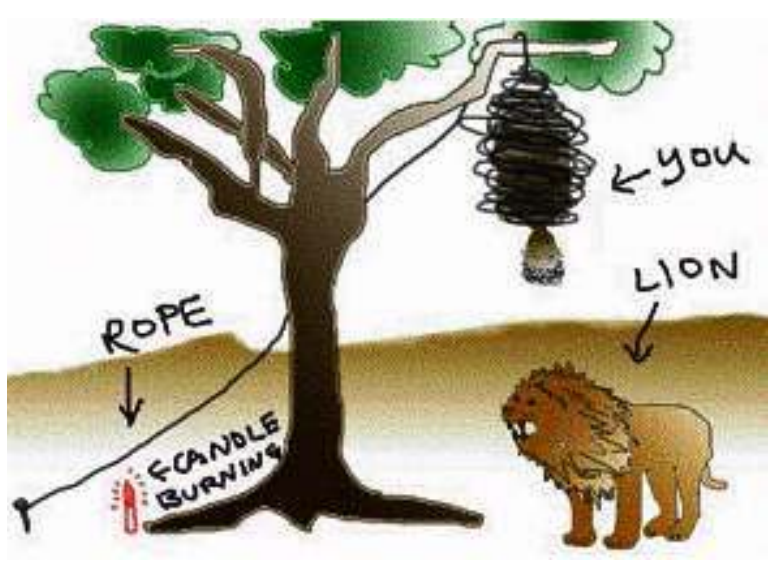

5 Sachant quevous êtes seul à des km à la ronde, que vous n'avez rien sur vous, 6 qu'allez vous donc faire?

6’ Aucune idée ? La solution plus bas...

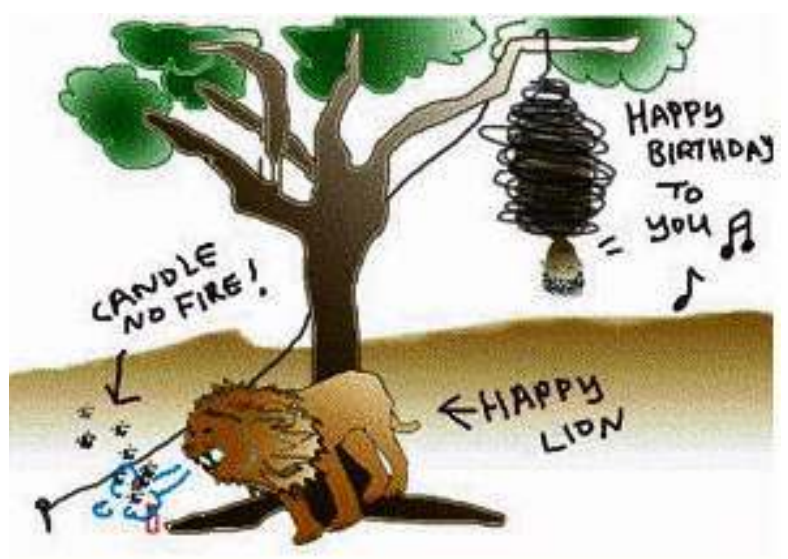

7 Vous chantez joyeux anniversaire au lion pour qu'il souffle la bougie.....

Soit :

\section{Situation générale}

Situation : 1

\section{Problème}

Problème : 2

Elaboration ${ }^{8}$ primaire du problème: $3,3^{\prime}$

Résumé de la situation problématique : $\mathbf{1}^{\mathrm{er}}$ dessin introduit par 4

Elaboration complémentaire du problème : 5

\section{Solution}

Perspective : 6, 6'

\section{Solution : 7 et $2^{\text {e }}$ dessin}

Sans anticiper sur la deuxième et la dernière partie de cet article, la $\mathrm{SE}^{9}$ est ici nominalement signalée : les noms situation et solution correspondent bien entendu, aux métatermes employés pour désigner certaines $\mathrm{SE}$, mais piège dans ce contexte ${ }^{10}$ est interprété comme problème (le début de l'histoire aurait pu être: Vous êtes en Afrique. Vous avez un petit problème: vous êtes attaché en haut d'un arbre...).Piège déclenche, autant 
qu'il signale, une SE. De même, idée renvoie à une proposition de solution. Il y a donc un réseau cohésif de noms qui balise le texte, et participe à sa structuration.

Les structures d'enchaînement se " coulent » dans les séquences textuelles pour profiler les textes de façons différentes selon les genres discursifs. Mais elles dominent également les relations de cohérence entre phrases. Dans l'exemple de la blague ci-dessus, 3 et 3' constituent pour 2 une relation d'élaboration à un niveau local. A un niveau plus global, ces mêmes phrases expriment la situation problématique. De même, au niveau interpropositionnel, vous chantez joyeux anniversaire au lion pour qu'il souffle la bougie est un énoncé articulé autour de la relation de cohérence cause / finalité. Mais on voit que cette articulation est particulièrement locale; d'un point de vue interprétatif, cet énoncé, exprimant la solution, est intégré à la SE. C'est en cela que l'on peut dire que cette dernière domine la relation de cohérence.

Un autre exemple emprunté à M. Charolles (1994) :

Exemple 3

- J'ai faim

- Passe moi le guide Michelin

où le type de relation discursive entre les deux interventions est difficile à définir. On peut voir, cependant, une cohérence prise en charge par le rapport [PROBLÈME - SOLUTION]. La référence, dans ce cas, à des implications contextuelles est sans doute légitime (ces implications seraient invoquées, par exemple, par la théorie de la Pertinence); mais on peut préférer voir l'effectivité pour les locuteurs d'un schéma non pas contextuel mais herméneutique, transposable dans des situations différentes. Dans ce petit exemple, et au contraire de l'exemple précédent, la SE n'est pas signalée, bien qu'elle soit efficiente au niveau interprétatif.

\section{Quelques propriétés des SE}

Les analyses des textes suivants permettent à la fois d'illustrer le fonctionnement des SE et de justifier la notion et le terme d'enchaînement.

\section{TEXTE 1}

1 La chute du Mur de Berlin et la désintégration de la sphère d'influence soviétique constituaient deux défis lancés à l'Union européenne. 2 L'une des réponses politiques apportées a été l'inscription de l'élargissement aux pays d'Europe centrale et orientale à l'ordre du jour de l'agenda européen. 3 Cinquante ans après le début de la construction européenne, l'élargissement à 25 membres et plus de l'Union européenne exige une adaptation de la structure institutionnelle et politique européenne.

http://www.ladocumentationfrancaise.fr/dossiers/constitutioneuropeenne/introduction.shtml

En 1, la chute du Mur de Berlin et la désintégration de la sphère d'influence soviétique sont deux situations évaluées comme problématiques (défis). 2 exprime la solution proposée (l'une desréponses). 3 expose une nouvellesituation problématique : l'élargissement à 25 membres et plus de l'Union européenne est reconnu comme problème qui demande une «solution ", une adaptation de la structure institutionnelle et politique européenne ${ }^{11}$. La suite du texte, non reproduite ici, expose les différentes étapes de cette adaptation; la principale est la signature le 29 octobre 2004, par les Etats membres, du Traité constitutionnel. L'étape finale, à venir au moment de l'élaboration de ce texte, est la ratification qui passe 
par les referenda. Autrement dit, l'une des étapes de la solution est civique et, d'une certaine façon discursive: dire oui ou non à la constitution. Pour cette raison, l'enchaînement en question, lorsque nous parlons de SE, peut être également intertextuel: la résolution d'un problème peut être apportée par un autre texte. Nos discours sont irrémédiablement enchainés les uns aux autres, grâce à leur performativité. Cette nature intertextuelle des SE est particulièrement saillante dans les emplois où problème et ses synonymes renvoient à des objets interdiscursifs : problème ou crise de la vache folle, de la pauvreté, problème palestinien, question des sans-papiers, etc. Ces expressions sont des «valises » transportées de discours en discours, qui se chargent d'un contenu toujours plus important.

Dans cette quatrième de couverture du roman policier Les égouts de Los Angeles de M. Connelly, la SE est beaucoup plus diffuse, mais non moins effective :

TEXTE 2

1 Né d'un père inconnu et d'une mère qui se prostituait, 2 l'inspecteur Harry (Hieronymus), Bosh - comme le peintre - voudrait bien oublier la guerre du Vietnam où il nettoyait des galeries souterraines creusées par la Vietcong.

3 Malheureusement pour lui, l'un de ces anciens collègues, Billy Meadows, a été assassiné dans une canalisation d'écoulement d'eaux de pluie d'Hollywood. 4 Le meurtre étant lié à une affaire de braquage, il faudra bien que, secondé et manipulé par la belle Eleanor Wish, agent très spécial du FBI, il affronte à nouveau sa peur.

Dans ce texte, 1 et $\mathbf{2}$ exposent une situation. A l'intérieur de celle-ci, est exprimé un objectif dont la réalisation ne suppose pas une action précise (il s'agit "simplement " d'oublier). 3 formule un problème double: un assassinat, qui constitue toujours une énigme (donc un problème) à résoudre pour un inspecteur. Et qui plus est, la victime est une personne connue de l'inspecteur - ce qui complique les choses! (cf. l'évaluatif malheureusement). La solution représentée par 4 passe par une épreuve (relativement stéréotypique et du conte, et de la fiction policière $\left.{ }^{12}\right)$ : affronter sa peur que l'on imagine liée aux galeries souterraines. Cette condition à la solution est considérée comme telle parce qu'opère la SE. En effet, le nœud « solution » n'est pas explicitement indiqué : il est seulement présupposé par la SE et alors identifié dans 4.4 pourrait être complétée par pour résoudre l'énigme: il faudra bien que, secondé et manipulé par la belle Eleanor Wish, agent très spécial du FBI, il affronte à nouveau sa peur pour résoudre l'énigme. Mais la proposition finale n'est pas utile: la cohérence interprétative du passage est imposée par la SE (typique du genre policier). Comme pour les relations de discours, les structures d'enchaînements sont parfois marquées, parfois non marquées.

Dans 4, une autre complication est annoncée, la manipulation par la belle Eleanor. Cette manipulation devra bien sûr trouver son dénouement dans le roman. Il y a donc ici imbrication de SE (OBJECTIF - RÉALISATION, PROBLÈME - SOLUTION, et récursivité de cette dernière $\mathrm{SE}$ ). Les $\mathrm{SE}$, de par leur plasticité, doivent être perçues comme des réseaux complexes, hiérarchisés, parfois amorcés puis avortés ${ }^{13}$, ou alors redistribués, recyclés, lorsque par exemple, une solution est évaluée négativement (cf. M. Hoey, 2000).

Enfin, dans ce courriel syndical reçu dans notre messagerie le 27 mars 2006 (nous laissons l'orthographe en l'état) :

TEXTE 3

Lors d'un interview donnée jeudi 23 mars 2006 sur France 3 Normandie et retransmise dans l'édition régionale du 19/20, la présidente de L'université de Caen Basse-Normandie affirme que les conditions de Sécurité commencent à être menacées dans les locaux occupés par les étudiants grévistes : "Les extincteurs ont 
été vidés, les issues de secours sont bloquées, les étudiants sont ivres une grande partie de la nuit". Cette situation, selon elle, pourrait justifier l'évacuation des locaux par les forces de police.

Pour les personnels de l'université, médiatiser principalement ce seul aspect de la situation des campus occupés ne vise qu'à jeter un discrédit sur l'ensemble du mouvement étudiant contre la précarité. Ce discours contribue à renforcer un état de tension en complète contradiction avec le dialogue que la présidente semble souhaiter.

Les personnels de l'université, présents jour et nuit sur les campus ont pu vérifier par eux-mêmes que cette présentation est une exagération caricaturale de phénomènes isolés, sans rapport avec la réalité du comportement de l'immense majorité des grévistes. Ceux-ci au contraire mettent tout en œuvre pour combattre les débordements éventuels.

Intersyndicale CGT, FSU, SUD

Il faut concevoir plusieurs SE en jeu dans ce texte. Toute SE est bien sûr inhérente à une position marquée dans le discours ; ainsi, on peut distinguer

La position « présidente de l'université »:

Situation (évaluée négativement) : dégradation (Les extincteurs ont été vidés, les issues de secours sont bloquées, les étudiants sont ivres une grande partie de la nuit)

Problème : menace des conditions de sécurité

Solution : éventuelle évacuation des locaux par les forces de l'ordre

La position " personnels de l'université » :

Situation (évaluée négativement) : prise de position de la présidente jetant un discrédit

Problème : renforcement de l'état de tension

\section{Solution : $\varnothing$}

La position " grévistes"

Situation : occupation des locaux

Problème : débordements éventuels

Solution : «mettre tout en œuvre » pour éviter les débordements

La SE de chaque position est bien sûr mise en scène par l'énonciateur collectif (l'intersyndicale); on ne saurait parler de SE objectives: elles sont construites, donc polémiques. La SE de la position « grévistes » est ramassée en une seule phrase, tandis que les deux autres, antagonistes, sont plus développées. Surtout, les SE "s'enchaînent " entre elles; on peut considérer, par exemple, que le nœud situation dans la SE de la position «personnels de l'université » est composé de l'intégralité de la SE de la position "présidente». En effet, la situation à partir de laquelle la tension s'échauffe entre dirigeants et personnels, est bien l'ensemble de l'articulation SITUATION - PROBLÈME SOLUTION considéré du point de vue de la présidente (tel que ce point de vue est construit par le discours). On peut même supposer que les solutions pour les uns soient des problèmes pour les autres; ainsi, la solution de la présidente, si elle devait se produire, serait incontestablement un problème pour les personnels de l'université (soutenant tous la grève, d'après le texte) et les grévistes. On voit donc les applications cognitives des multiples SE dans un texte: l'interprétant doit être capable de repérer les différentes positions et de leur associer leur propre $\mathrm{SE}$, et de là, prêter aux participants des intentionnalités propres. Même si un point de vue idéologique domine, en principe celui 
de l'énonciateur, plusieurs façons d'appréhender des situations sont présentes, qu'elles soient discréditées ou non par l'énonciateur.

Les structures d'enchaînements sont donc des modes d'organisation de l'expérience et des discours, dont les diverses formes ne sont pas dénombrées, mais qui peuvent être énoncées par les paires PROBLÈME - SOLUTION, DÉSIR - ASSOUVISSEMENT, OBJECTIF - RÉALISATION, etc., sachant que ces paires sont complétées par d'autres nœuds comme situation et évaluation ${ }^{14}$ . Chaque actualisation des structures d'enchaînement ne donne pas lieu à une réalisation complète : la solution peut ne pas être évoquée, la situation initiale ne pas être exprimée, etc. Le terme même d'enchaînement se justifie à plusieurs titres :

- enchainement des sujets au monde : ces structures ont une dimension cognitive, sémiotique et herméneutique qui marque un rapport fondamental à l'expérience ;

- enchaînement des sujets entre eux : chaque nœud anticipe sur un autre nœud. Autrement dit, les tensions créées par le texte obligent l'énonciateur à satisfaire les attentes de l'énonciataire ;

- enchaînement des phrases et parties du textes entre elles ;

- enchaînement des SE liées à des positions différentes ;

- enchaînement des textes à d'autres textes : la résolution d'un conflit peut être apportée par un autre texte.

S'il n'est pas question d'affirmer que tout texte se déploie selon la logique des structures d'enchaînement, il convenait seulement, dans cette partie et à partir de quelques exemples, de montrer la fonctionnalité de ce mode d'organisation dont l'efficience doit mieux être appréciée. Dans les parties suivantes, nous donnons les éléments nécessaires à la détermination d'une certaine catégorie nominale ayant pour fonction, entre autre, de signaler dans le texte certaines SE.

\section{Des noms à la recherche d'un contenu ${ }^{15}$}

\section{Introduction}

61 Dans certains des textes étudiés dans la première partie, les SE sont lexicalement signalées par des unités comme : situation, danger, problème, énigme, piège, solution, réponse. On peut affirmer que ces noms appartiennent à une catégorie identifiable grâce à des critères syntaxiques mis en évidence par Vendler (1968) pour l'anglais, mais facilement adaptables au français. Ainsi, il existe des items nominaux pouvant être employés dans les constructions avec constituants phrastiques :

- 1- $\mathrm{N}$ être de inf. : l'objectif est de négocier avec les syndicats

- 1 '- $\mathbf{N}$ être que $\mathbf{P}$ : le problème est que le premier ministre ne veut pas négocier

Ces constructions connaissent très souvent une variante, plus « orale $»^{16}:$ la présence $d u$ pronom de reprise. Ainsi, on entend très souvent à l'oral une forme comme

Le problème, c'est d'arriver à négocier

63 Les énoncés avec pronom de reprise sont proches des constructions pseudo-clivées dans leur dispositif de thématisation / focalisation retardée ; surtout, par rapport aux énoncés sans pronom de reprise, ils marquent de façon plus prononcée la constitution interactionnelle des textes, autrement dit leur dimension dialogale :

2' - quel est le problème?

- c'est d'arriver à négocier 

peuvent être employés dans l'une ou l'autre des structures (ou dans les deux) - sans comptabiliser les formes nominalisées d'adjectifs (le mieux, l'important, l'essentiel, l'emmerdant est de participer). Comme nous le verrons plus loin, ces constructions sont assez proches dans leur fonctionnement et leur nature des pseudo-clivées.

\section{Caractérisation des constructions spécificationnelles}

Dans ce travail, nous nous intéressons aux constructions spécificationnelles pour deux raisons : 1) elles constituent le critère d'identification des noms sous-spécifiés (autrement dit, seule cette catégorie intègre la construction). 2) elles possèdent des propriétés discursives évidentes que nous discutons plus loin.

La grammaire "traditionnelle" recense des constructions à sujets réels et à sujets apparents. De la même façon, on peut considérer qu'il y a ici un attribut ${ }^{17}$ apparent, en fait sujet réel : l'infinitif ou la complétive précédé du complémenteur ; le GN serait alors un sujet apparent, et un attribut réel :

3- (de) négocier avec les syndicats est l'objectif

3'- que le premier ministre ne veuille pas négocier est le problème.

68 L'inversion du rapport sujet - attribut opère à la fois une thématisation (serait posé comme thème, un objectif, un problème, une solution, une question, etc.), et une focalisation sur l'élément rhématique (amplifiée car retardée lorsqu'il y a pronom de reprise).

On parlera ici de construction au sens des Grammaires de Construction ${ }^{18}$ (en particulier Goldberg, 1995, 1998), c'est-à-dire qu'il existe un appariement entre une forme syntaxique et un emploi soit sémantique, soit pragmatique particulier ${ }^{19}$ (à l'image, par exemple, de la phrase clivée qui partage d'ailleurs la même fonction de focalisation). Ce type de construction est désigné ici par le terme construction (ou énoncé) spécificationnelle, terme inspiré par certains travaux de la Grammaire Générative (Higgins (1979), Ruwet (1983)) qui parlent précisément de phrases à copule dites «spécificationnelles »; ainsi Ruwet (1983), propose les exemples :

4- le problème est que Luc a menti à Paul ; mon plus cher désir est d'être présenté à Luc; un gros défaut de Jules est son amour immodéré pour la boisson; mon meilleur ami est Paul.

Pour Ruwet et Higgins, le sujet de ces phrases n'est pas référentiel (pas plus que l'élément l'attribut) : il présente une variable dont l'attribut spécifie une valeur. On remarquera que mon meilleur ami est Paul ne répond pas aux critères évoqués :

* mon meilleur ami est de inf /que $P$

72 Phrases à copules spécificationnelles et constructions spécificationnelles ne se recouvrent donc pas.

\section{Les noms sous-spécifiés}

73 Entre donc dans les constructions spécificationnelles, une catégorie nominale désignée ici par l'étiquette noms sous-spécifiés ${ }^{20}$; la sous spécification en question est d'ordre sémantico-conceptuel : il est en effet difficile de se représenter un objet prototypique 
d'une classe dénommée par le nom (quel est le prototype, par exemple, de la classe désignée par les mots objectif,réponse?). Autrement dit, ces noms relativement « abstraits » (mais comptables), ne donnent pas naissance à des représentations précises ou stables des objets auxquels ils renvoient virtuellement ${ }^{21}$. La sous spécification trouve généralement une "solution" dans le texte qui généralement spécifie le contenu (la valeur) du nom - ce que Winter (1992) nomme la réalisation lexicale. Ainsi les exemples :

5- Lucie a eu une idée (emprunté à C.Schnedecker (2004: 267), qui observe le phénomène).

Cette phrase souffre d'incomplétude en raison de la sous spécification du nom idée; on s'attend donc à ce que le texte vienne combler un vide informationnel.

6- Il y a une différence entre la gauche et la droite

Cet énoncé, de la même façon, est incomplet, et demande un étayage : différence déclenche le besoin d'une explication.

Les constructions spécificationnelles constituent donc, à un certain niveau d'analyse, un critère d'identification des noms sous-spécifiés. Ici idée et différence entreraient dans une structure spécificationnelle :

5'- L'idée de Lucie est de connaître les intentions de Paul

6'- La différence est que la gauche peut mobiliser les syndicats

Si seuls les noms sous-spécifiés connaissent ce type d'emplois, ils peuvent évidemment être employés dans d'autres configurations, comme l'anaphore résomptive par exemple, ou encore, pour certains d'entre eux dans les formes « N que P » / « N de Inf. » (le fait que, l'hypothèse que... ${ }^{22}$. Les noms pouvant être employés dans ces dernières formes sont nommés noms à compléments propositionnels par M. Riegel (1996) dont les observations peuvent être en partie généralisées à la catégorie englobante des noms sous-spécifiés.

\section{Caractéristiques des noms sous-spécifiés}

Un travail conséquent pour l'anglais (et dont nous nous inspirons ici) a été mené par H.G. Schmid (2000). L'auteur nomme shell nouns les noms qui répondent aux critères de Vendler (nos noms sous-spécifiés) ; la métaphore de la coquille se justifie par le fait que ces noms semblent transporter avec eux un contenu conceptuel, qui ne leur est pas inhérent, et qu'ils peuvent facilement abandonner pour se mouvoir de façon libre. En adoptant les propos de Schmid (qui se fonde lui-même sur Ivanič (1991) et Conte (1996)), et en les complétant, il est possible de distinguer trois comportements concomitants : un comportement mi-pronominal, mi-nominal, un comportement de modalisateur, et un comportement de connecteur.

\section{Comportement mi-pronominal, mi-nominal}

Ces noms possèdent les propriétés syntaxiques des noms en général (modifications adjectivales ou par relatives), expriment une signification propre - leur signification lexicale - à partir de laquelle aucune représentation mentale n'est véritablement possible (en raison de cette signification générale, abstraite). Mais ils acquièrent une dimension plus « substantielle » en contexte, grâce au contenu véhiculé par la nominalisation dans les structures décrites plus haut, ou bien par un contenu du texte non adjacent. Ils établissent donc à la fois une relation à l'expérience stable décrite par le contenu lexical (comme les noms typiques) mais reçoivent une détermination de leur contenu contextuellement à l'image des pronoms (le mot chose analysé par Kleiber (1987), nom 
sous-spécifié, a , comme on le sait, été utilisé comme pronom dans un certain état de la langue,). Ce sont donc des variables auxquelles le cotexte plus ou moins élargi apporte une valeur : ces noms « souffrent » donc d'une sorte d'incomplétude sémantique.

Par ailleurs, les noms non-spécifiés forment ce que le psychologue Barsalou (1983) nomme des catégorisations ad hoc. Selon certaines approches en linguistique cognitive, l'idée de formation de concepts est utile pour le traitement de l'information, mais participe en fait d'une illusion créée par la langue, pour deux raisons : on a l'impression que le mot vaut pour une entité simple, au contour défini (empaquetage ou encapsulationConte (1996)) et que l'entité possède une substance (réification ou hypostase). L'empaquetage de ces informations dans des concepts nominaux temporaires permet d'avoir l'illusion de frontières conceptuelles nettes et rigides, et donc d'avoir l'illusion de manipuler des concepts eux-mêmes bien définis. C'est une sorte d'illusion référentielle, un effet de concrétisation construit par métaphore grammaticale dans le sens de Halliday (1996) et relevant également de la métaphore du conduit de M.Reddy (1979) : les mots sont perçus comme des contenants (ou des coquilles).

Dans l'extrait suivant :

\section{TEXTE 4}

Comment rendre accessibles les milliards de pages HTML qui peuplent la Toile aux nouveaux terminaux? Epineux problème. Car ce qu'il est pertinent d'avoir à l'écran d'un ordinateur de bureau n'a bien souvent que peu de rapport avec ce qu'il est utile - voire possible - de rapatrier depuis un PDA (Personal Digital Assistant, ou assistant personnel numérique), un téléphone mobile, un téléviseur ou un Minitel. Tailles d'écran variables, systèmes d'exploitation différents, il est nécessaire de garder le sens des documents, mais d'en changer la forme.(Le Monde 17 janvier 2001, Langages XML : une nébuleuse en expansion).

Epineux problème est une prédication extraphrastique portant sur le co-texte gauche, constituant une méta-énonciation (les noms sous-spécifiés affectionnent ce type d'emplois:question cruciale, chose étonnante, fait surprenant, souvent en construction détachée). Le syntagme est employé en tout début de texte, c'est-à-dire à un moment clé : l'introduction du topique est directe. Ivanič, Conte et Schmidont remarqué le placement stratégique de ces noms; ainsi, on les repère non seulement en début de texte, mais également en toute fin pour signaler, par exemple, qu'un problème ou une question devra être traité dans le futur - norme à laquelle contribue abondamment l'exercice scolaire de la rédaction et de la dissertation (ouvrir, dans la conclusion, une autre problématique).

Par sa signification lexicale, problème caractérise l'information antécédente. Le nom problème est bien sûr évaluatif, mais son contenu se trouve lui-même objet d'évaluation ( épineux). Ainsi, le nom sert-il de support à la prédication adjectivale. Cette évaluation ne pouvait porter directement sur le contenu du problème :

TEXTE 4 '

* Comment rendre accessibles les milliards de pages HTML qui peuplent la Toile aux nouveaux terminaux est épineux.

\section{Bien sûr l'entame}

\section{TEXTE 4"}

Rendre accessibles les milliards de pages HTML qui peuplent la Toile aux nouveaux terminaux est épineux.

était possible; mais la configuration du texte changeait alors quelque peu: perte de l'allure dialogale (disparition de l'interrogative), et signalisation du problème retardée à la fin de la phrase par l'attributif. Au lieu de cela, l'entame originale correspond à un 
certain canon de l'écriture journaliste que reconnait d'emblée le lecteur : la question à résoudre. Dans l'interrogative, au contraire de TEXTE 4", le problème est signalé dès le début de la phrase :

Comment rendre accessibles $\rightarrow$ comment faire ( pour que $\mathrm{X}$ soit $\mathrm{Y})$ ? ce que je crois, c'est que $P$ (les pseudo-clivées ; cf. M.N. Roubaud, 2000, pour une analyse très précise du phénomène) constitue un modalisateur. Il rapproche alors ces énoncés des séquences avec thème détaché ; ainsi :

7- Chose remarquable et qui prouve que l'esprit humain n'est pas naturellement si farouche et révolté qu'on le croit communément, c'est que les premiers essais de la liberté, ses plus hardies tentatives au moyen âge consistaient à s'immoler, à se perdre en Dieu (Michelet, Journal, ex. emprunté à Furukawa, 2005, p.162).

7'- Ce qui est remarquable et qui prouve que l'esprit humain n'est pas naturellement si farouche et révolté qu'on le croit communément, c'est que les premiers essais de la liberté, ses plus hardies tentatives au moyen âge consistaient à s'immoler, à se perdre en Dieu (transformation de Furukawa, 2005, p.162).

7"- Leremarquable et qui prouve que l'esprit humain n'est pas naturellement si farouche et révolté qu'on le croit communément, c'est que les premiers essais 
de la liberté, ses plus hardies tentatives au moyen âge consistaient à s'immoler, à se perdre en Dieu.

Si Ivanič relèvent le fonctionnement pronominal des shell nouns, ils metten également en évidence leur rôle d'organisateur textuel (mettre en rapport des parties du texte) sans pourtant faire référence explicitement à leur aspect «connecteurs ». Or, les noms sous-spécifiés, surtout lorsqu'ils sont employés dans une construction spécificationnelle, font souvent office de connecteurs, et peuvent alors marquer plusieurs relations discursives entre phrases du texte. Quelques exemples :

Relation d'explication (causale)

10- Je n'étais qu'un petit sot ; aussi étais-je très tranchant. Je donnais toujours tort à mon parrain. Le fait est qu'il n'employait pas des termes éblouissants comme son adversaire. (A. France, La vie en fleur, 1922).

Relation d'énumération

11- Cette réclusion où elle était tenue, elle me l'avoua avec franchise, pour diverses raisons. La principale est qu'elle s'imaginait, bien que je n'eusse écrit que des articles ou publié que des études, que j'étais un auteur connu, ce qui lui faisait même naïvement dire, se rappelant. (M. Proust, Le temps retrouvé, 1922).

Relation contrastive ${ }^{24}$

12- Sans ces chaînes la pauvreté ne serait pas la souffrance et elle perdrait peut-être tout mérite. Que demanderait-on au monde? Un peu d'argent et la liberté. Le malheur est qu'il ne peut pas vous donner ensemble l'un et l'autre, et que l'un est trop souvent le prix de l'autre. Une parcelle infime de telle substance guérit, une forte dose tue. (P.Reverdy, Le gant de crin, 1927).

Nous présentons une analyse détaillée à partir du texte suivant :

TEXTE 5

La Constitution brésilienne de 1988 impose la protection des propriétés indigènes, un processus comprenant pas moins de 11 à 14 étapes. Le PPG 7 (programme de conservation de la forêt tropicale) " a permis d'accélérer le mouvement ". Actuellement, $440000 \mathrm{~km}^{2}$ de terres sont en cours de régularisation (soit $8 \%$ de la 
forêt). A terme, $10 \%$ de la surface de la forêt amazonienne devraient être transformés en réserves naturelles, suivant un accord entre le WWF et le gouvernement brésilien. Néanmoins, les pays donateurs pointent les différents dysfonctionnements qui freinent la réalisation du programme: objectifs trop vastes, absence de connexions entre les différents projets, complexité des montages financiers, blocages internes et coûts administratifs excessifs.

L'optimisme résiste malgré tout, s'appuyant sur le fait que $85 \%$ de la forêt amazonienne sont encore intacts. " On sait qu'il y a de quoi mettre en place un développement durable de l'Amazonie. Le problème est que cela entre en conflit avec les autres politiques de développement du gouvernement brésilien ", explique Hervé Théry. Le ralentissement de la croissance démographique devrait freiner le mouvement pionnier et une prise de conscience de l'intérêt de protéger un capital naturel aussi fabuleux se développe chez les Brésiliens.(Le Monde,31 janvier 2001, page 29)

Dans cette position thématique, le nom sous-spécifié est figé en syntagme, grammaticalisé avec l'article défini cataphorique (le singulier est contraint), dans sa fonction de mise en opposition.

Le cotexte gauche de l'énoncé spécificationnel exprime une évaluation positive, que met en contraste la valeur droite du nom. "Problème» thématise ce contraste, tout en opérant une focalisation - un peu à l'image d'une clivée - sur la proposition qui suit, permettant de diriger en quelque sorte l'attention du lecteur de façon beaucoup plus efficace qu'une phrase non marquée comme :

\section{TEXTE 5'}

cependant, cela entre en conflit avec les autres politiques de développement du gouvernement brésilien

où il y a bien également contraste, nécessairement signalé par un connecteur, mais sans marquage de focalisation sur la proposition. Dans cet emploi, «problème », comme on l'a vu, emprunte autant au fonctionnement nominal que pronominal, mais il emprunte également aux connecteurs leur fonction de signalement de relation de cohérence.

Par ailleurs, le TEXTE 5' oblige à uncoût cognitif élevé pour établir la relation de cohérence avec ce qui suit (le ralentissement de la croissance démographique devrait freiner le mouvement pionnier ...). On s'attend en effet, suite à cette évaluation, à une explication du conflit (autrement dit, un étayage de la valeur du «shell noun»). Or, cette phrase ((le ralentissement de la croissance démographique ...) exprime un contenu constituant une solution éventuelle au problème (on aurait pu avoir : Cependant, le problème peut se résoudre parle ralentissement de la croissance démographique qui devrait freiner...). Dans le texte, la " solution », comme complémentaire, n'est certes pas signalée, mais elle est anticipée par le nom problème, ce qui fait que, malgré l'attente déçue, on établit sans trop de difficultés, en tout cas plus facilement, la relation PROBLÈME - SOLUTION.

Notons enfin, à un niveau strictement énonciatif, que l'énoncé spécificationnel est employé dans du discours direct ${ }^{25}$; il ne s'agit pas là d'un épiphénomène. Au contraire, ce trait est caractéristique de la rédaction journalistique. Dans Legallois et Richard (à par.), nous avons examiné que cet emploi est fréquent, et surtout, qu'il est systématique lorsque problème est modifié par le modal vrai (le vrai problème est que...); sans considérer la structure avec infinitif, ni la construction avec pronom de reprise, l'examen de quatre années du journal Le Monde (de janvier 1999 à décembre 2002) ne permet certes de relever que dix occurrences ${ }^{26}$, mais sur ces 10 occurrences, 9 sont localisées dans du discours direct. 
104 Les comportements des noms spécifiques sont donc multiples; entre pronoms et noms, modalisateurs, connecteurs, ils constituent, lorsqu'ils sont employés dans les structures spécificationnelles, des moyens expressifs spectaculaires, capables de cumuler plusieurs propriétés en même temps. Ce travail n'étant qu'un premier éclairage, il est possible que d'autres propriétés peuvent surgir à l'analyse. Dans la partie suivante, nous mettons un peu plus en évidence ce que certains points de la partie 2 sous-entendaient: la connivence entre noms sous-spécifiés et SE. Autrement dit, nous nous intéressons aux fonctionnements textuels des noms sous-spécifiés lorsqu'ils signalent des SE.

\section{Les noms sous-spécifiés « marqueurs »}

\section{Introduction}

Le rapport entre constructions spécificationnelles et noms sous-spécifiés étant établi, il est nécessaire maintenant de préciser celui qui existe entre SE et noms sous-spécifiés.

Nous avons observé dans la première partie que les SE sont des schémas ou des routines herméneutiques valables pour tout type de textes. Elles ne donnent pas nécessairement lieu à un signalement spécifique ; cependant, les métatermes utilisés pour les désigner sont employés parfois comme simples noms dans les discours; ce sont des noms sousspécifiés : situation, problème, question, réponse, solution, objectif, désir, etc. peuvent être employés dans les énoncés spécificationnels. Tous les noms sous-spécifiés ne signalent évidemment pas des SE; seuls certains d'entre eux le font, soit dans les constructions spécificationnelles, soit dans d'autres constructions. L'ensemble des noms sous-spécifiés signalant une SE sera tout simplement dénommé classe des noms sous-spécifiés marqueurs.

\section{Analyse}

107 En l'état de nos travaux, nous ne pouvons dresser une liste définie des noms sousspécifiés marqueurs; aussi, l'étude que nous proposons consiste seulement à observer, à partir d'un texte, le fonctionnement de trois de ces items, sans pouvoir évidemment proposer de généralisation définitive.

108 Le texte étudié est donné dans sa quasi-intégralité, et cela malgré l'analyse locale que nous proposons. L'intérêt du texte réside paradoxalement dans son aspect relativement fastidieux et technique ${ }^{27}$; les noms sous-spécifiés marqueurs, qui signalent ici une SE PROBLÈME - SOLUTION, permettent justement de faciliter la tâche de rédaction, celle de la lecture, et celle de l'interprétation.

TEXTE 6

Vers une remise en cause de la politique agricole commune des Quinze.

La " révolution " agricole allemande se heurte aux réalités du marché

Regagner la confiance des consommateurs- électeurs en parant au plus pressé

Cela a été un réflexe de survie. En appelant à " en finir avec les fabriques agricoles

", en transformant le ministère de l'agriculture en un ministère de la protection des consommateurs, le chancelier Gerhard Schröder a renversé la vapeur, alors que le pays sombrait dans l'hystérie depuis la découverte du premier cas de vache folle, en novembre 2000. Le ministre de l'agriculture, le social-démocrate Karl-Heinz Funke, et celle de la santé, la Verte Andrea Fischer, ont été sacrifiés le 9 janvier. En confiant l'agriculture aux Verts, à une fille de la ville, la Berlinoise Renate Künast, Gerhard Schröder espérait réitérer un de ces " coups " dont il a le secret. 
Le coupable a été désigné : le lobby agricole. " Le syndicat des agriculteurs doit tabler sur une baisse de son influence sur la politique agricole ", a prévenu M. Schröder, ajoutant que celle-ci serait pensée " du point du vue du consommateur ". Les Allemands se sont déclarés au trois quarts favorables à l'idée d'accorder des aides en fonction de critères écologiques.

Le gouvernement n'a pas encore présenté de concept très précis, ni analysé toutes les conséquences de ce virage. Mme Künast doit présenter ses idées devant le Parlement en février. Pour l'heure, le pays modifie ses habitudes alimentaires - un tiers des Allemands mangent moins de boeuf, un quart n'en consomment plus -, les cours et la consommation s'effondrent. Mme Künast veut faire passer de 2 à $20 \%$, en dix ans, la part des produits de l'agriculture biologique dans l'alimentation. L'objectif paraît peu réaliste aux experts. " Les Allemands préfèrent dépenser leur argent dans leur voiture, leurs habits ou leurs vacances ", affirme l'hebdomadaire Die Woche.

Aujourd'hui, seuls 8,4\% des Allemands citent le prix comme argument d'achat de la nourriture, mais reste à savoir si cette affirmation est durable et surtout se vérifie. En dépit de la crise, $70 \%$ d'entre eux n'ont pas acheté plus de produits bio qu'auparavant. Le potentiel d'amélioration existe: seulement $1,8 \%$ des exploitations agricoles allemandes sont bio, contre $8 \%$ en Autriche et $0,5 \%$ en France. Le commissaire européen à l'agriculture, Franz Fischler, a noté que l'Allemagne n'utilisait pas les possibilités de la politique agricole commune (PAC) pour réorienter une partie des aides agricoles vers l'agriculture biologique.

Second problème, le bio doit, selon les lois du marketing, rester un produit de niche pour être rentable. S'il atteignait une part de marché de $20 \%$, il s'imposerait comme norme de consommation, et l'agriculture traditionnelle serait obligée de l'adopter. Ceci reviendrait à élever toutes les normes en Allemagne, ce qui a un coût. Le consommateur acceptera-t-il de le payer, surtout si des produits de même qualité sont disponibles hors de l'Union?

[...]

Que peut faire l'Allemagne pour se protéger? La logique voudrait qu'elle demande de réintroduire des barrières tarifaires ou augmente ses subventions à l'agriculture. Ces deux solutions vont à l'encontre du sens des négociations de l'OMC et promettent un bel affrontement avec les Etats-Unis, ce qui va vite agacer les industriels allemands. La seconde est, de surcroît, en opposition avec les positions défendues jusqu'à présent par le chancelier Schröder et son ministre des affaires étrangères Joschka Fischer : en période de vaches maigres, ils sont exaspérés par la PAC, qui engloutit la moitié du budget européen.

[...]

HYPOTHÈSE PROVOCATRICE La dernière solution serait d'accepter que l'Allemagne - et l'Europe - est mal placée pour produire des biens agricoles, et qu'en application de la division internationale du travail il vaut mieux laisser ce marché à des pays comme l'Argentine, plus compétitifs. Hypothèse provocatrice, qui reviendrait à casser quarante ans de politique européenne, fondée sur l'idée de développer une agriculture non compétitive dans un continent alors en déficit alimentaire. Ce serait aller au clash avec la France mais aussi, en Allemagne, avec la Bavière, qui estime que ses agriculteurs font partie de son patrimoine culturel.

Ces questions se poseront, en termes plus subtils, si l'Allemagne pousse jusqu'au bout sa logique. Mais, comme dans le nucléaire, où la sortie de l'atome a été très progressive, il n'est pas exclu qu'après l'effet d'annonce elle adopte une politique des petits pas, plus pragmatique et moins source de conflits. Il est trop tôt pour en juger. Pour regagner la confiance des consommateurs-électeurs, on pare au plus pressé, proposant des mesures pour renforcer la lutte contre l'épizootie, comme la généralisation des tests sur les animaux de deux ans. Mais, en cette fin de semaine, l'émotion avait lieu en Saxe-Anhalt, à l'est. Une vache infectée a été trouvée dans une ferme géante héritée de la RDA : mille bêtes doivent être conduites à l'abattoir. (Le Monde, 29 janvier 2001 - Arnaud Leparmentier) 
De façon évidente, second problème permet de catégoriser ce qui précède comme premier problème. Il s'agit d'une homologation indirecte, dont l'effet est la formation d'une prédication présupposée, non exprimée, en quelque sorte in absentia ; la portée de second problème est donc double : portée à droite, bien sûr, comme prédicat anticipé. Et portée à gauche, par catégorisation présupposée par l'adjectif. Le nom sous-spécifié sert d'ailleurs de support à ce marqueur de sériation. Mais ces remarques ne doivent pas masquer une fonction qui nous semble spectaculaire ici. Le caractère problématique des faits rapportés dans les premiers paragraphes était déjà saillant, et ne nécessitait pas, de ce fait, une caractérisation par le nom. Donc, l'intérêt de l'emploi est ailleurs : c'est le phénomène d'empaquetage qui est là encore fondamental. Si l'on veut déterminer le contenu précis du premier problème, on rencontre quelques difficultés. Les quatre premiers paragraphes exposent en effet, non pas un problème, mais un ensemble de problèmes, certes liés entre eux, mais en fait de natures différentes. Ainsi, on peut énumérer : crise de la vache folle, crise politique, perte de l'influence des syndicats, dictature des consommateurs, effondrement des cours, stagnation du marché bio, indifférence aux possibilités de la PAC. Soit, une série de "complications ", subsumée sous la tutelle du nom sous-spécifié, empaquetée en un tout. La complexité de cette série est en quelque sorte effacée d'un trait, ou d'un mot: il y a instruction pour considérer les premiers paragraphes comme constituant une seule unité textuelle. Nous ajoutons: les problèmes sont de natures différentes, mais surtout, ils émanent de plans différents; ainsi, l'hystérie de la vache folle, l'effondrement des cours, stagnation du marché sont des problèmes «factuels ", constatables. En revanche, faire passer de $2 \grave{a} 20 \%$, en dix ans, la part des produits de l'agriculture biologique dans l'alimentation signalé comme problème (l'objectif paraît peu réaliste)ne relève pas à proprement parler du factuel, mais d'une intention, plus exactement d'un programme. Les deux ordres sont donc confondus et unifiés sous la même étiquette, assimilés dans la même hypostase. Les SE sont présentes dans ces premiers paragraphes; en effet aux problèmes sont associées parfois des solutions locales (par ex. accorder des aides en fonction de critères écologiques / utiliser les possibilités de la politique agricole). Mais ces réponses ne sont que mentionnées, ou brièvement évaluées. Les « véritables » solutions que propose le texte seront présentées au sixième paragraphe (la logique voudrait que [l'Allemagne]) demande de réintroduire des barrières tarifaires)... en réponse à la question globale que peut faire l'Allemagne pour se protéger?

Second problème permet d'ouvrir un second mouvement en ménageant une relation avec le cotexte gauche : il y a à la fois continuité (on reste dans les problèmes), et instauration d'une nouvelle dynamique informative, qu'une expression comme " par ailleurs, le bio doit, selon les lois du marketing... » ne pourrait impulser aussi efficacement. Là encore, il est utile de se poser la question de la nature de ce second problème: il s'agit en fait d'une anticipation, ou d'une projection ; plus exactement, on pose le caractère problématique de la réalisation du programme politique (faire passer de 2 à $20 \%$, en dix ans, la part des produits de l'agriculture biologique), lui-même évalué comme...problématique (peu réaliste) dans le troisième paragraphe. Le second problème est donc une extension, un étayage (un paragraphe " pour lui tout seul») d'une composante du premier problème. De ce fait, les deux problèmes ne sont objectivement pas à mettre sur le même plan. La sériation qu'opère le syntagme avec le nom sous-spécifié permet la mise en marche d'une mécanique paradoxale: globalisation / nivellement d'un ensemble de complications, détachement d'un étayage d'une de ces complications pour sa mise en relief, catégorisation de ces deux unités dans la même classe. La fonction d'hypostase tourne à 
plein, pour permettre de lancer un peu plus loin la question résumante que peut faire l'Allemagne pour se protéger?

111 L'expression ces deux solutions ${ }^{28}$ caractérise indirectement la question précédente comme problème ou objectif, et permet un partitionnement de l'information bien commode : une deuxième puis une dernière solution sont développées ou commentées. Avec le pronom «cela » (cela va à l'encontre...) il y avait une conjonction parfaite des «solutions", qui empêchait de marquer la différence, la spécificité de chacune. L'étayage textuel développant la "seconde solution ", une relation satellite au sens de Mann et Thompson (1986), aurait été beaucoup plus difficile à introduire et aurait obligé à exprimer à nouveau le contenu.Ce partitionnement (1, 2, 3 solution) est doublé par un autre : solution 1 et 2 sont des solutions logiques, naturelles (mais évaluées comme problématiques) tandis que la dernière solution est perçue comme hypothèse provocatrice (donc également problématique - hypothèse est un nom sous-spécifié en emploi thématique dans les deux occurrences). Grâce à la répétition du mot «solution » qui crée un lien de cohésion, la relation de confrontation, assez complexe ici parce qu'elle donne lieu à la fois à un contraste (logique / provocation) et à une similitude (les deux types de solutions engendrent d'autres problèmes - encore le jeu de l'enchevêtrement des SE), est assez facilement reconnue. Sans noms sous-spécifiés, sans hypostase, ces relations, pour être exprimées, obligeraient à une gymnastique rédactionnelle et à une lecture acrobatique.

Enfin,ces questions se poseront, en termes plus subtils, anaphore résomptive, nivelle à son tour l'imbrication des SE PROBLÈME - SOLUTION, pour à nouveau évoquer, dans un cadre hypothétique, une solution (mauvaise) de l'Allemagne - adopter une politique des petits pas.

Le texte illustre donc une participation active des noms sous-spécifiés marqueurs à la structuration globale des discours; cette structuration ne se limite pas à une simple connexion entre parties de textes. On l'a vu, nivellement de l'hétérogène, partitionnement, contraste, remise à zéro du compteur thématique sont ainsi élaborés à partir des fonctions connecteur et hypostase, ainsi que par le jeu des co-occurrences des noms sous-spécifiés marqueurs : problème, logique, solutions, hypothèse, questions forment un réseau lexical cohésif et structurant, signalant des SE et les remodelant à la fois.

\section{En conclusion}

Ce travail avait pour objectif de porter un premier regard sur deux phénomènes complémentaires, encore peu évalués par la linguistique. Certes, à sa manière, la didactique a souvent considéré les catégories telles que PROBLÈME - SOLUTION comme articulations de certains discours, mais il revient à la linguistique textuelle d'en examiner les marqueurs implicites ou explicites. En cela, une grammaire des constructions spécificationnelles, en se développant à partir des caractéristiques mises en évidence ici, doit apporter des éléments nécessaires à une analyse précise des faits de constructions textuelles. Elle permettra en outre de mieux apprécier le rôle des noms sous-spécifiés, dont la liste devra constituer la base, non pas d'un classement, mais de plusieurs classements possibles selon l'analyse du comportement privilégié : indexicalité, modalité, connexité. Observer ces noms en vertu de leur(s) «fonctionnement(s) » oblige à considérer en quelque sorte, pour suivre Hunston et Francis (2000:185), une "nouvelle » classe de mots dont la nature ne peut se résumer aux seules propriétés nominales « traditionnelles » : substantives, référentielles abstraites/ concrètes. 


\section{BIBLIOGRAPHIE}

ADAM J.M. (1992) Les textes: types et prototypes, Nathan université

BARSALOU L. (1983), “Ad hoc categories” in Memory and Cognition, 11:211-227

BERRENDONNER A. (2005) « Question et mémoire discursive », in Rossari, Beaulieu-Masson, Cojocariu et Razgouliaeva, Les états de la question, Québec : Editions Nota Bene : 146-169

BREMOND Cl. (1973) Logique du récit, Éd. du Seuil

CHAROLLES M. (1994) « Cohésion, cohérence et pertinence du discours », in Travaux de Linguistique 29, pp. 125-150

CONTE M-E (1996) « Anaphoric encapsulation » in De Mulder Walter \& Tasmowski Liliane (eds) Coherence \& anaphorahttp://www.benjamins.nl/cgi-bin/t_bookview.cgi?bookid=BJL\%2010 Belgian Journal of Linguistics, 10

CORNISH Francis (à paraître 2006) «Relations de cohérence et anaphores en contexte inter-phrastique: une symbiose parfaite» in Dominique Legallois, Unité(s) du texte, Langages 164

FRANCIS G. (1994) Labelling discourse: an aspect of nominal-group lexical cohesion 83-101, Coulthard, Malcolm, ed., Advances in Written Text Analysis, (London and New York: Routledge

FURUKAWA N. (2005), Pour une sémantique des constructions grammaticales : Thème et thématicité, Duculot

GOLDBERG A.E, 1995, Constructions : A Construction Grammar Approach to Argument Structure, University of Chicago Press

GOLDBERG A.E., 1998, «Patterns of experience in patterns of language». The new psychology of language cognitive and functional approaches to language structure, edited by M. Tomasello, Lawrence Erlbaum Associates, Publishers, Mahwah, New Jersey, London : 203-219

GREIMAS AJ (1983), Du Sens II, Paris, Seuil

GREIMAS A. J. (1966), Sémantique structurale, Paris, Larousse

HALLIDAY M. (1996) An introduction to functional grammar, Sydney : Arnold

HIGGINS F. (1979), The pseudo-cleft construction in English, New York, Garland

HOEY M. (1983) On the Surface of Discourse. London: George Allen \& Unwin

HOEY M. (1994) "Signalling in discourse: a functional analysis of a common discourse pattern in written and spoken English" in Coulthard, Malcolm, ed., Advances in Written Text Analysis, London and New York: Routledge, 26-45

HOEY M. (2000) Textual Interaction: An Introduction to Written Discourse Analysis, Routledge

HUNSTON S. and FRANCIS G (2000), Pattern Grammar, John Benjamins

HUNSTON S. and THOMPSON G. (eds.) (2000) Evaluation in text: authorial stance and the construction of discourse. Oxford, Oxford University Press

HUTCHINS J. (1977) “On the structure of scientific texts”, UEA Papers in linguistics 5, 18-39 
IVANIČ Roz (1991) « Nouns in search of a context » in RAL XXIX / 2 : 93 - 114.

JOLLES A. (1972) Formes simples, Paris, Éd. du Seuil

JORDAN M.(2001) "Some discourse patterns and signalling of the assessment-basis relation" in Scott, Mike and Geoff Thompson, eds. Patterns of Text: In Honour of Michael Hoey, John Benjamins

KEHLER Andrew (2002) Coherence, Reference, and the Theory of Grammar, CSLI Publications

KLEIBER G. (1987) « Mais à quoi sert donc le mot CHOSE ? Une situation paradoxale », Langue française, 73, pp. 109-127.

LEGALLOIS \& J. FRANÇOIS (2006) Autour des grammaires de constructions et de patterns, Cahier du CRISCO 21 http://www.crisco.unicaen.fr

LEGALLOIS D. (à par.) « Du bon usage des expressions idiomatiques dans l'argumentation de deux modèles anglo-saxons : la Grammaire de Construction et la Grammaire des Patterns »

LEGALLOIS D. et FERRARI S. (à par.) « Vers une grammaire locale de l'évaluation pour l'analyse du discours ", Actes du colloque "Discours et Documents », Caen juin 2006

LEGALLOIS D. et RICHARD E. (à par.) « Vrai problème/véritable problème » et autres questions à propos de la grammaire compositionnelle et de la grammaire fondée sur l'usage », Actes du colloque Aux marges des grammaires - hommage à Michèle Noailly, Presses Universitaires de Rennes.

MANN W.C. \& THOMPSON S. (1986) : « Rhetorical Structure Theory: a theory of text organization", in The Structure of Discourse, L. Polanyi, Norwood, N.J.:Ablex

MARTIN J. \& WHITE P. (2005), The Language of Evaluation: Appraisal in English, Palgrave Macmillan Hardcover.

REDDY M. (1979) The conduit metaphor - a case of frame conflict in our language about language. In A. Ortony, editor, Metaphor and Thought, pages 284-324. Cambridge University Press, Cambridge.

RIEGEL Martin (1996)« Les noms à compléments propositionnels : en quoi sont-ils plus abstraits que les autres? » Les noms abstraits : histoire et théories, actes du colloque de Dunkerque, 15-18 septembre 1992 ; textes réunis par Flaux, Glatigny, Samain, Villeneuve-d'Ascq, Presses universitaires du Septentrion : 313-321

ROUBAUD M.N. (2000) Les constructions pseudo-clivées en français contemporain, Paris, Honoré Champion

RUWET N. (1983) « Du bon usage des expressions idiomatiques dans l'argumentation en syntaxe générative » Revue québécoise de linguistique 13.

SCHMID H.J. (2000) English Abstract Nouns as Conceptual Shells: From Corpus to Cognition, Walter de Gruyter.

SCHNEDECKER C. (2004) «Particularités référentielles et (macro) syntaxiques des textes à double ou à "multi-topique". Description linguistique et perspectives cognitives » in S. Porhier et D. Klinger, L'unité Texte, Association Perspectives.

STERN D. (2005) «L'enveloppe prénarrative : Vers une unité fondamentale d'expérience permettant d'explorer la réalité psychique du bébé », Bernard Golse, Sylvain Missonnier (éds) Récit, attachement et psychanalyse. Pour une clinique de la narrativité, ERES

VENDLER Z.(1968) Adjectives and nominalizations, Mouton

WINTER E. (1992) "The Notion of Unspecific versus Specific as one Way of Analysing the Information of a Fund-Raising Letter". In Mann, William C. and Sandra A. Thompson (eds.) 
Discourse Description: Diverse linguistic analyses of a fund-raising text. Pragmatics \& Beyond, New Series, Amsterdam, John Benjamins.

WINTER E. (1994) "Clause relations as information structure: two basic text structures in English," Coulthard, Malcolm, ed., Advances in Written Text Analysis, London and New York: Routledge

\section{NOTES}

1. Cf. Francis Cornish (2006) et ici même, Mann et Thompson (1986), Kehler (2002).

2. D'autres désignations, comme celle de plan d'organisation, ont parfois été données.

3. La sémantique des genres et des types oblige nécessairement (et souvent salutairement) à une disjonction entre «natures » textuelles; il faudrait, cependant, qu'en parallèle, soit discriminée « la nature fondamentale » du texte, c'est-à-dire ce qui fait que n'importe quel texte est un texte.

4. Il faudrait bien sûr mettre en rapport les notions de SE et de formes simples d'A. Jolles (1972).

5. Les travaux britanniques ont en revanche prêté une certaine attention à ces structures: Winter (1994), Hoey (2000), Jordan (2001).

6. Les SE sont rarement binaires, malgré ce que pourrait laisser entendre leur notation (il y a le plus souvent adjonction d'une situation ou d'une évaluation). Les termes employés ici sont des métatermes. Problème, par exemple, peut très bien renvoyer à des unités linguistiques telles que pépin, ennui, accident, catastrophe, guerre, manque à gagner, etc.

7. Nous numérotons les différentes articulations de la SE.

8. Elaboration s'entend ici comme « détaillement» (cf. Kehler, $2002: 18$ ).

9. Que l'on pourrait représenter certainement autrement. Par exemple, on indiquant la hiérarchie entre situation générale (être en Afrique) et situation problématique (être pris dans un piège en Afrique).

10. L'interprétation piège = problème est contextuelle. Imaginons la même situation mais d'un autre point de vue : le piège est la solution (le moyen) pour satisfaire le sacrifice rituel (objectif à réaliser) d'une tribu.

11. Segment qui constitue d'ailleurs un lien hypertexte vers une autre page du site: la page quelle constitution? Il est tout à fait significatif, ici, que ce soit un « nœud » de la SE qui soit choisi comme « lien » vers un complément textuel.

12. On pense bien sûr également à Vertigo d'Hitchcock.

13. Tout problème ne trouve pas sa solution, tout objectif n'est pas nécessairement réalisé. Surtout, un texte peut évoquer explicitement une situation problématique, sans que soient développées systématiquement des réponses.

14. Sans développer la notion d'évaluation, il convient d'en dire quelques mots dans cette note : l'aspect évaluatif dans la SE est tout à fait fondamental. La difficulté est que l'évaluation intervient à deux niveaux qui ne sont pas à confondre : elle est bien un nœud particulier dans la structure, lorsqu'elle constitue à elle seule une étape: par exemple, la morale d'une histoire forme bien une évaluation, de même que la conclusion d'une explication. Mais l'évaluation est effective à un niveau bien plus fondamental : la complication ou le problème, comme il a été vu, sont le fruit d'une évaluation; on juge ou estime problématique une situation, de même que l'on considère comme solution tel programme. En cela, l'évaluation ne saurait constituer un nœud particulier. Parce qu'elle instaure un rapport fondamental au monde, l'évaluation traduit en termes euphorique / dysphorique les situations dans lesquelles nous sommes jetés, et permet l'émergence du sens et de la rationalité. Citons Greimas : "Une catégorie sémantique peut être axiologisée par la projection, sur le carré qui l'articule, de la catégorie thymique dont les termes contraires sont dénommés / euphorie / vs / dysphorie /. Il s'agit d'une catégorie "primitive ", 
dite aussi proprioceptive, à l'aide de laquelle on cherche à formuler, très sommairement, la manière dont tout être vivant, inscrit dans un milieu, "se sent " lui-même et réagit à son environnement, un être vivant étant considéré comme un système d'attractions et de répulsions. » (Greimas, 1983 : 93). Notons que la linguistique anglo-saxonne développe un grand nombre de travaux sur la question de l'évaluation: Hunston et Thompson (2000), Jordan (2001), Martin et White (2005) ; voir également Legallois et Ferrari (à paraître)

15. Ou, pour reprendre le titre de R. Ivanič (1991), Nouns in search of a context

16. Mentionnons également une autre variante: La logique voudrait que l'Allemagne demande de réintroduire des barrières tarifaires.

17. Attribut s'entend ici davantage dans une acception syntaxique que sémantique : l'élément à droite ne caractérise pas l'élément gauche, mais l'identifie par spécification.

18. Ou encore de patterns (Hunston et Francis, 2000). Cf. Legallois et François (2006).

19. "A construction is [...] a pairing of form with meaning/use such that some aspect of the form or some aspect of the meaning/use is not strictly predictable from the component parts or from other constructions already established to exist in the language" (A. Goldberg, 1998, 205)

20. Winter (1992), parle d' unspecific nouns. Précisons dans cette note que noms sous-spécifiés renvoie plutôt à un type d'emploi nominal caractérisé par des comportements qu'à une véritable catégorie à part.

21. Comme le remarque Winter (1992), ce sont de très mauvais candidats pour les métaphores.

22. Notons encore les formes : l'hypothèse consiste à..., l'idée selon laquelle...la solution réside dans le fait que....Nous avons entrepris un travail d'identification et d'analyse des constructions " sœurs » des constructions spécificationnelles

23. En partie à cause de la relative coordonnée.

24. Furukawa (2005) relève pour les énoncés spécificationnels du type «ce que je crois, c'est que... » une relation discursive de rupture - ce que nous appelons de façon peut être plus appropriée, relation de contraste, ou d'opposition. Il n' y a généralement pas de rupture dans le développement du topique général.

25. Cette remarque est valable non pas pour tous les noms sous-spécifiés, mais seulement pour problème.

26. Dans la plupart des constructions avec vrai, l'attribut-valeur est constitué par un SN, un adjectif ou une forme « de + infinitif ».

27. A moins que l'on ne ressente un intérêt particulier pour la politique agricole allemande.

28. Précédé de l'énoncé spécificationnel la logique voudrait qu'elle demande de réintroduire des barrières tarifaires. L'emploi de logique est fortement cohésif ici avec solutions et hypothèse. Il a un rôle indéniable de modalisateur.

\section{RÉSUMÉS}

Les séquences d'enchaînement constituent un moyen d'organisation textuelle intermédiaire entre les relations de cohérence et les séquences textuelles. Ces structures, particulièrement polymorphes et récursives, se réalisent dans des schémas de type situation-évaluation / problème-solution / objectif-réalisation, etc. Herméneutiquement et dialogiquement motivées, elles sont signalées à l'interprétant par des moyens lexico-grammaticaux. Je m'intéresserai, dans certains emplois, aux fonctionnements syntaxique, sémantique, textuel de noms «sous- 
spécifiés » comme problème, question, solution, réponse (désignés par le terme de shell nouns dans la terminologie britannique), signalant explicitement des structures d'enchaînement. Ces noms, employés ou non dans la construction spécificationnelle du type « $\mathrm{N}$ est de +INF » / « N est que $+\mathrm{P} »$, forment une classe particulière empruntant ses caractéristiques aussi bien à la catégorie nominale «typique » qu'à la catégorie pronominale et à celle des connecteurs

"Linking sequences" are cohsive devices by which some texts are organised; for example, the well-kwnown problem-solution schema is a general principle that expains the semantic connection within as well as between the clauses of a text. These patterns are often signaled by a category of "unspecific nouns" which can take a nominalisation as complement in the construction "N-be-that ..." (idea, problem, solution, question, etc.). We illustrate in this paper the characteristics and functions of both "linking sequences" and "unspecific nouns".

\section{INDEX}

Mots-clés : structures d'enchaînement, noms sous-spécifiés, problème-solution, cohérence textuelle, hypostase

Keywords : linking sequences, shell nouns, problem-solution, text coherence, hypostasis

\section{AUTEUR}

DOMINIQUE LEGALLOIS

CRISCO (CNRS - FRE 2805) - Université de Caen 